\title{
Does domperidone, a dopamine 2 receptor-antagonist, alter gastric emptying rates in healthy adults?
}

\author{
O. Markey and A. Shafat \\ Department of Physical Education and Sport Sciences, University of Limerick, Limerick, Republic of Ireland
}

Domperidone (Motilium ${ }^{\mathrm{TM}}$, Janssen-Cilag Ltd., UK) is a peripheral dopamine 2 receptor-antagonist with upper gastrointestinal tract prokinetic activity and anti-emetic properties ${ }^{(1)}$. This drug is commonly used to alleviate postprandial nausea, abdominal bloating and early satiety in patients with diabetes and dyspepsia as well as healthy adults. The interaction of fat with the small intestine delays gastric emptying rate (GER) resulting in the suppression of appetite and energy intake ${ }^{(2)}$. Faster gastric emptying may lead to hyperlipidaemia which in turn can significantly impair vascular function after a single high-fat meal ${ }^{(3)}$. The present study aimed to determine if acute domperidone administration (i) accelerates GER of a high-fat solid meal and (ii) alters satiety sensations in healthy volunteers.

Nine healthy volunteers $\{4$ males and 5 females, age 25.3 (SD 5.0) years, weight 74.9 (SD 12.8) kg, height 1.76 (SD 0.12 ) $\mathrm{m}$ and BMI 24.2 (SD 2.9) $\mathrm{kg} / \mathrm{m}^{2}$ \} consented to participate in the single blind, randomized placebo-controlled cross-over study, which was approved by the University of Limerick Research Ethics Committee. After fasting overnight, volunteers ingested Motilium ${ }^{\mathrm{TM}}$ ( $10 \mathrm{mg}^{\mathrm{momperidone})}$ or matched placebo pill 30 minutes before a high-fat pancake breakfast meal (3625 kJ (866 kcal); \% energy: 44 from fat, 44 from carbohydrate, 12 from protein) incorporating $100 \mathrm{mg}\left[{ }^{13} \mathrm{C}\right]$ octanoic acid. Postprandial breath samples were collected every $5 \mathrm{~min}$ during the first 30 minutes and thereafter at $15 \mathrm{~min}$ intervals for $6 \mathrm{~h}$. Analysis of the ${ }^{13} \mathrm{CO}_{2}$ excretion curve provided estimates of gastric emptying half time, lag phase, latency and ascension time ${ }^{(4,5)}$. A visual analogue scale (VAS) identified changes in satiety during the test period. Statistical analysis was by paired $t$-tests or two-way ANOVA with between (treatment) and within (time) subjects, with significance at $P<0.05$.

No significant differences were found between gastric emptying variables under placebo and drug conditions; however a strong trend did exist for accelerated half time and lag phase (Table 1). VAS data illustrated that domperidone did not affect satiety sensations $(P>0.05)$.

Table 1. Gastric emptying time variables under placebo and drug conditions

\begin{tabular}{lccccc}
\hline Time (min) & Placebo & SD & Drug & SD & $P$ \\
\hline Half time & 239 & 58 & 212 & 49 & 0.07 \\
Lag Phase & 136 & 31 & 125 & 24 & 0.06 \\
Latency time & 40 & 11 & 39 & 11 & 0.98 \\
Ascension time & 197 & 53 & 174 & 49 & 0.10 \\
\hline
\end{tabular}

This study illustrates that domperidone has a strong tendency to accelerate the GER of a high-fat breakfast in healthy adults, with no detectable effect on satiety sensations. Endothelial dysfunction is one of the earliest incidents in the development of CVD ${ }^{(3)}$. Domperidone may increase postprandial lipaemia by accelerating the delivery of fats into the small intestine. Further investigation is needed to examine the effects of domperidone on food intake and vascular health.

1. Xu DH, Lou HG, Yuan H, Jiang B, Zhou Q, Zhang ZM \& Ruan ZR (2008) Biomed Chromatogr 22, 433-440.

2. Little TJ, Horowitz M \& Feinle-Bisset C (2007) Am J Clin Nutr 86, 531-541.

3. Tsai W-C, Li Y-H, Lin C-C, Chao T-H \& Chen J-H (2004) Clin Sci 106, 315-319.

4. Ghoos YF, Maes BD, Geypens BJ, Mys G, Hiele MI, Rutgeerts PJ \& Vantrappen G (1993) Gastroenterology 104, $1640-1647$.

5. Schommartz B, Ziegler D \& Schadewaldt P (1998) Isotopes Environ Health Stud 34, 135-143. 